\title{
TRIPLE-NEGATIVE BREAST CARCINOMAS AS TUMORS UNTRACEABLE BY CONVENTIONAL RADIOLOGICAL METHODS: A RETROSPECTIVE COHORT
}

Vanessa Monteiro Sanvido', Morgana Domingues da Silva', Patricia Zaideman Charf¹, Gil Facina', Afonso Celso Pinto Nazário'

${ }^{1}$ Universidade Federal de São Paulo - São Paulo (SP), Brazil.

Introduction: Invasive breast carcinoma represents a heterogeneous group of lesions that differ in their molecular and histological characteristics. Perou et al. evaluated breast tumors using the DNA microarray technique and classified them into four molecular subtypes: Luminal A (LA), Luminal B (LB), HER2 overexpression (HER2), and triple-negative (TN). Immunohistochemistry approximately identifies the subtypes. The TN subtype is negative for estrogen and progesterone receptors and HER 2 protein. This subgroup is comprehensive, with $75 \%$ of them being basaloid, that is, cells with a molecular profile similar to that of myoepithelial cells and a high expression 5, 6, 14, and 17 cytokeratins, vimentin, and P-cadherin. These tumors tend to be more aggressive, have higher rates of cell proliferation, and, therefore, a worse prognosis. Clinically, triple-negative carcinomas are more strongly associated with younger patients, early local and distant recurrence. Given their rapid progression, they can be clinically diagnosed in the interval of screening tests. Objective: To compare clinical and radiological aspects of TN and other molecular subtypes of breast cancer at diagnosis. Method: The study retrospectively evaluated data collected from medical records of patients diagnosed with breast cancer and treated at the Hospital São Paulo from 2013 to 2016. Results: In the study period, 235 cases of breast cancer were diagnosed. The incidence in patients under 39 years was $4.2 \%$ for LA, $4.9 \%$ for LB, and $8.3 \%$ for TN. At diagnosis, $83 \%$ of patients with TN tumors had clinical complaints, of which $96 \%$ were nodules. In mammographies, TN presented as nodules in $100 \%$ of cases, LA in 68\%, LB in 71\%, and HER2 in 50\%. Microcalcifications were identified in 14\% of LA cases, $21 \%$ of LB, and 50\% of HER2. TN had no cases of microcalcifications or asymmetries. Among the other subtypes, the diagnosis by physical examination represented $35 \%$ to $53 \%$ of cases. As to the staging at diagnosis, TN cases presented $\leq 2 \mathrm{~cm}$ tumors in $25 \%$ of cases. The LA, LB, and HER2 subtypes presented as $\leq 2 \mathrm{~cm}$ tumors, respectively, in $61 \%, 49.4 \%$, and $43 \%$ of patients. Lymph node involvement by neoplasm at diagnosis occurred in 3.35\%, 17.5\%, 14.3\%, and 33.3\% of LA, LB, HER2, and TN cases, respectively. Conclusion: TN carcinomas affect a greater number of young patients, outside the screening age group. In our sample, TN tumors were diagnosed based on clinical complaints and showed no association with non-palpable breast lesions. TN is the subtype with the highest probability of interval tumors, untraceable by conventional exams, and, as a result, other screening options, such as serum assays, have been discussed. 